\title{
Inhibition of activated Ras suppresses multiple oncogenic Hub genes in human epithelial tumors
}

\author{
LEI CAO $^{1,2^{*}}$, PING WANG $^{3 *}$, HUI LUO $^{1 *}$, XI-RUI WANG ${ }^{1 *}$, XIE-FENG WANG $^{1}$, JUN-XIA ZHANG $^{1}$, \\ YING-YI WANG $^{1}$, LEI YAO ${ }^{1}$, NING LIU ${ }^{1}$ and YONG-PING YOU ${ }^{1}$ \\ ${ }^{1}$ Department of Neurosurgery, The First Affiliated Hospital of Nanjing Medical University, Nanjing 210029; \\ ${ }^{2}$ Department of Neurosurgery, The Affiliated Xuzhou Hospital of Southeast University, \\ Xuzhou 221009; ${ }^{3}$ Liver Transplantation Center, The First Affiliated Hospital \\ with Nanjing Medical University, Nanjing 210029, P.R. China
}

Received December 16, 2013; Accepted June 10, 2014

DOI: $10.3892 /$ ijo.2014.2532

\begin{abstract}
Cancer cells may involve diverse mutations, but they often rely on continued expression of a single oncoprotein for survival, as a response to targeting this protein. Generally, Ras is overexpressed in human epithelial tumors and cancellation of activated Ras inhibits carcinoma cell proliferation and differentiation ability, and induces apoptotosis of tumor cells. However, the mechanisms of inhibition of activated Ras that suppress the malignancy activity of human epithelial tumors remain to be illuminated. We utilized text-mining of MEDLINE abstracts with natural language processing to establish the Ras biologic association network, and identified several interactions of this network with the Ras pathway. Our investigation not only examined the expression of Ras and Hub genes (PIK3CA, MDM2, CCND1, EGFR, JUN, MYC, VEGFA, ERK1 and ERK2) but also confirmed inhibition of activated Ras reduced expression of multiple oncogene in vitro studies. Our studies provide strong support for the conclusion that cancellation of activated Ras specifically regulates defective Ras pathways in human tumor cells.
\end{abstract}

\section{Introduction}

Malignant tumors are the major malignancy cousing threat to human health. The conventional treatments of cancer include tumorectomy, radiotherapy and chemotherapy. However, these treatments are not effective in curing cancer, or improving the prognosis or lowering the mortality (1). Molecular biology

Correspondence to: Dr Y.P. You or Dr N. Liu, Department of Neurosurgery, The First Affiliated Hospital of Nanjing Medical University, Nanjing 210029, P.R. China

E-mail: yyp19@njmu.edu.cn

E-mail: liuning0853@yahoo.com.cn

${ }^{*}$ Contributed equally

Key words: glioma, bioinformatics, RAS, signaling pathway has advanced greatly, yet there is still no ultimate solution for the biological behavior of malignant tumors, which include unlimited proliferation, differentiation and invasion ability (2).

Ras gene, as a human oncogene, was the first to be identified in the viral oncogenes of Harvey and Kirsten murine sarcoma viruses (3). Subsequently, three distinct transforming genes were discovered, and they were present in human-tumor cell lines designated $\mathrm{H}, \mathrm{K}$ and $\mathrm{N}$-ras $(4,5)$, respectively. The Ras protein of transcription and translation might regulate a variety of biological functions including cell growth, differentiation and death (6). In addition, previous studies showed that tumor malignancies were linked to the Ras protein. However, the mechanisms remain largely undetermined causing the effects of Ras on cancer cells.

In this study, to investigate the underlying mechanisms of repression of activated Ras downregulation tumor cell growth, we extracted gene/protein interactions by bioinformatics and constructed the Ras biologic association network. We confirmed Ras and Hub genes expression in glioma, lung cancer and bladder cancer tissues and inhibition of activated Ras led to repression of U87, T24 and A549 human epithelial tumor cell proliferation and apoptosis induction in vitro. Bioinformatics analysis revealed an association of the Ras network with the Ras signaling pathway. Furthermore, we discovered that downregulation of Ras inhibited several members of this pathway in human epithelial tumor cells.

\section{Materials and methods}

Text mining (NLP analysis). To carry out bioinformatics text mining, we used MEDLINE as the literature source. We queried MEDLINE with: (N-ras or K-ras or H-ras or ras) and (cancer or tumor) and ("1980/01/01"[PDAT]: "2010/02/01"[PDAT]). The abstracts were downloaded as documents and organized into XML format. Genes mentioned (including the ras gene) were tagged using ABNER. Moreover, we prepared to conjunction resolution, for example the STAT3/5 gene was anatomised STAT3 gene and STAT5 gene. Because of the plethora of gene variant names, all gene names were normalised based on Entrez database (http://www.ncbi.nlm.nih.gov/Entrez/) to facilitate 
test and comparison. Owing to the higher frequency of the gene, there can be more correlation with some disease. In the end, we collected and analyzed the information for each gene.

Next, $\boldsymbol{m}$ and $\boldsymbol{n}$ deputized for the frequency of gene mentions. $\boldsymbol{k}$ was the number of Gene-Disease simultaneously. The number of literature we searched in Pubmed represented $N$. The hypergeometric distribution test was employed.

$$
p=1-\sum_{i=0}^{k-1} p(i \mid n, m, N)
$$

Where:

$$
p(i \mid n, m, N)=\frac{n !(N-n) ! m !(N-m) !}{(n-i) ! i !(n-m) !(N-n-m+i) ! N !}
$$

Gene ontology analysis. In this experiment, our genes mapped to each node of gene ontology database and the number of genes in these nodes were computed. The software package of GSEABase in the R Project for Statistical Computing (http:// www.r-project.org/) was used. Finally, differentially expressed genes were classified according to three independent modes such as biological process, cellular component and molecular function.

Pathway and gene network analysis. We used GenMAPP v2.1 (GenMAPP - Home Page) to make our genes mapped to KEGG pathway database and to calculate the enrichment p-value of gene in each pathway. We downloaded the pathway data in KEGG database, and used KEGGSOAP software package (http://www.bioconductor.org/packages/2.4/bioc/ html/KEGGSOAP.html) which was in the R Project (http:// www.r-project.org/) to make an assay of the interaction of genes genome-wide, included three connection: ECrel (enzyme-enzyme relation, indicating two enzymes catalyzing successive reaction steps), PPrel (protein-protein interaction, such as binding and modification), GErel (gene expression interaction, indicating relation of transcription factor and target gene product). Considering the results of the three data sets, we integrated it as the network of mutual relations between genes.

Cell culture and treatment. Human U87 glioma cell line, T24 bladder cancer cell line and A549 lung cancer cell line, purchased from the Chinese Academy of Sciences Cell Bank, were grown in DMEM (Gibco USA) and 1640 (Gibco) supplemented with $10 \%$ fetal bovine serum, $100 \mathrm{U} / \mathrm{ml}$ penicillin and $100 \mu \mathrm{g} / \mathrm{ml}$ streptomycin. The cells were incubated at $37^{\circ} \mathrm{C}$ in a humidified atmosphere of $95 \%$ air and $5 \% \mathrm{CO}_{2}$. All cell lines were plated at $10^{7}$ cells per well in 6-well plates and our experiment was separate into three groups: blank group, negative control group treated with DMSO and intervention group treated with FTS (Ras inhibitor, Farnesyl Thiosalicylic Acid, Santa Cruz, USA).

Tumor specimens. Our study was approved by the Medical Review Board of Nanjing Medical University. All tissue samples were obtained from The First Affiliated Hospital of Nanjing Medical University. Forty-five human glioma tissues, including 15 grade II tumors, 15 grade III tumors, and
15 grade IV tumors (GBM), were obtained from patients undergoing surgery for glioma in the Department of Neurosurgery. In addition, ten normal brain tissues were collected as a negative control from patients undergoing decompressive craniectomy for traumatic brain injury. Fifteen lung tissues, 15 adjacent non-cancer tissue and 15 lung cancer tissue samples were from the Department of Chest Surgery, and 15 adjacent noncancer tissue and 15 bladder cancer tissue samples were from the Department of Urinary Surgery. All cancer tissue samples were resected during surgery and immediately frozen in liquid nitrogen for subsequent total RNA extraction.

RT-PCR analysis. All RNA were extracted from three tumor tissues using TRIzol reagent (Invitrogen) according to the standard protocol, and converted to cDNA by a PCR-kit (AMV) Ver.3.0 (Takara). PCR reactions were proceeded with using EZuse Taq polymerase (Generay Biotech, Shanghai, China). In addition, we performed PCR conditions as follows: $95^{\circ} \mathrm{C}$ for $30 \mathrm{sec}, 58^{\circ} \mathrm{C}$ for $30 \mathrm{sec}$ and $72^{\circ} \mathrm{C}$ for $60 \mathrm{sec}$ for 30 cycles. The primer sequences are listed in Table I. The PCR products for the gene were electrophoresed on a $2 \%$ agarose gel stained with ethideum bromide. Gels were scanned with Gel Doc ${ }^{\mathrm{TM}}$ XR gel documentation system (Bio-Rad).

Cell proliferation assay. To examine relative cell growth, the MTT assay was prepared. Cells were plated at $10^{4}$ cells per well in 96-well plates, treated with different doses $(25,50,75$ and $100 \mu \mathrm{M})$. The number of viable cells was determined with MTT reagent (Keygen, China) post-treatment. The cell viability was measured at $540 \mathrm{~nm}$ absorbance using an enzyme-linked immunosorbent assay plate reader. The percent viability was calculated considering the controls as $100 \%$. All data points represent the mean of a minimum of six wells.

Western blot analysis. The total protein was obtained from three human cancer cell lines using RIPA (Applygen, Beijing, China) and the concentration was determined by BCA method (Keygen, China). The lysate was subjected to SDS-PAGE in 10 or $12 \%$ acrylamide gel and then transferred to PVDF membranes (Millipore Corp., USA). The membranes were obturated in defatted milk. Next the protein were subjected to immunoblot analysis with one of the following antibodies: anti-PIK3CA, MDM2, CCND1, EGFR, JUN, MYC, VEGFA, ERK1 and ERK2 (Santa Cruz, 1:1,000 dilution).

Apoptosis analysis. The cells were plated in 6-well plates and treated with FTS. After $48 \mathrm{~h}$, according to the manufacturer's instructions, the apoptosis ratio was assayed using Annexin V-FITC Apoptosis Detection kit (BD Biosciences, San Diego, CA, USA) with flow cytometry. Annexin $\mathrm{V}^{-}$and $\mathrm{PI}^{-}$cells were used as controls. Annexin $\mathrm{V}^{+}$and $\mathrm{PI}^{-}$cells were considered as early apoptotic and Annexin $\mathrm{V}^{+}$and $\mathrm{PI}^{+}$cells as late apoptotic and necrotic cells.

Cell cycle analysis. Forty-eight hours after treatment, cells in the log phase of growth were harvested, washed with PBS for three times and fixed with $70 \%$ ethanol at $-20^{\circ} \mathrm{C}$ for $1 \mathrm{~h}$. In addition, next after PBS washing, the cells were suspended in $50 \mathrm{mg} / \mathrm{ml}$ propidium iodide (PI) (Sigma-Aldrich) and $20 \mathrm{mg} / \mathrm{ml}$ RNaseA (Boehringer-Mannheim), incubated for 
Table I. Primer sequences.

Primers

\begin{tabular}{|c|c|c|}
\hline Gene & Up & Down \\
\hline 1 Ras & GGGGTTTGGTCTCTGTGCCAGC & GCACTGCAGTTCCTGAAGTATGGC \\
\hline 2 PIK3CA & GGAGCCTGGAAGAGCCCCGA & ACACACTGGCATGCCGATAGCA \\
\hline 3 MDM2 & TTTCGCAGCCAGGAGCACCG & CCATCCGGGGTTTTCGCGCT \\
\hline 4 CCND1 & AGAGGGCTGTCGGCGCAGTA & TTTGCAGCAGCTCCTCGGGC \\
\hline 5 EGFR & TGCGAAGGCGCCACATCGTT & TACACCGTGCCGAACGCACC \\
\hline $6 \mathrm{JUN}$ & GCACCTCCGCGCCAAGAACT & TCAGCGCGCCTGGGTTGAAG \\
\hline $7 \mathrm{MYC}$ & ACGGCCGACCAGCTGGAGAT & TGGGCGAGCTGCTGTCGTTG \\
\hline 8 VEGFA & TCGGGCCTCCGAAACCATGA & CCTGGTGAGAGATCTGGTTC \\
\hline 9 ERK1 & TGGCAGGCAGGCAGGCAATC & GGCCCCACGTCGAACACCTG \\
\hline 10 ERK2 & GCTGCGCTTCCGCCATGAGA & GGTGGAGCACGTTGGCGGAG \\
\hline $11 \beta$-actin & AAGACCTGTACGCCAACACAGT & AGAAGCATTTGCGGTGGACGAT \\
\hline
\end{tabular}
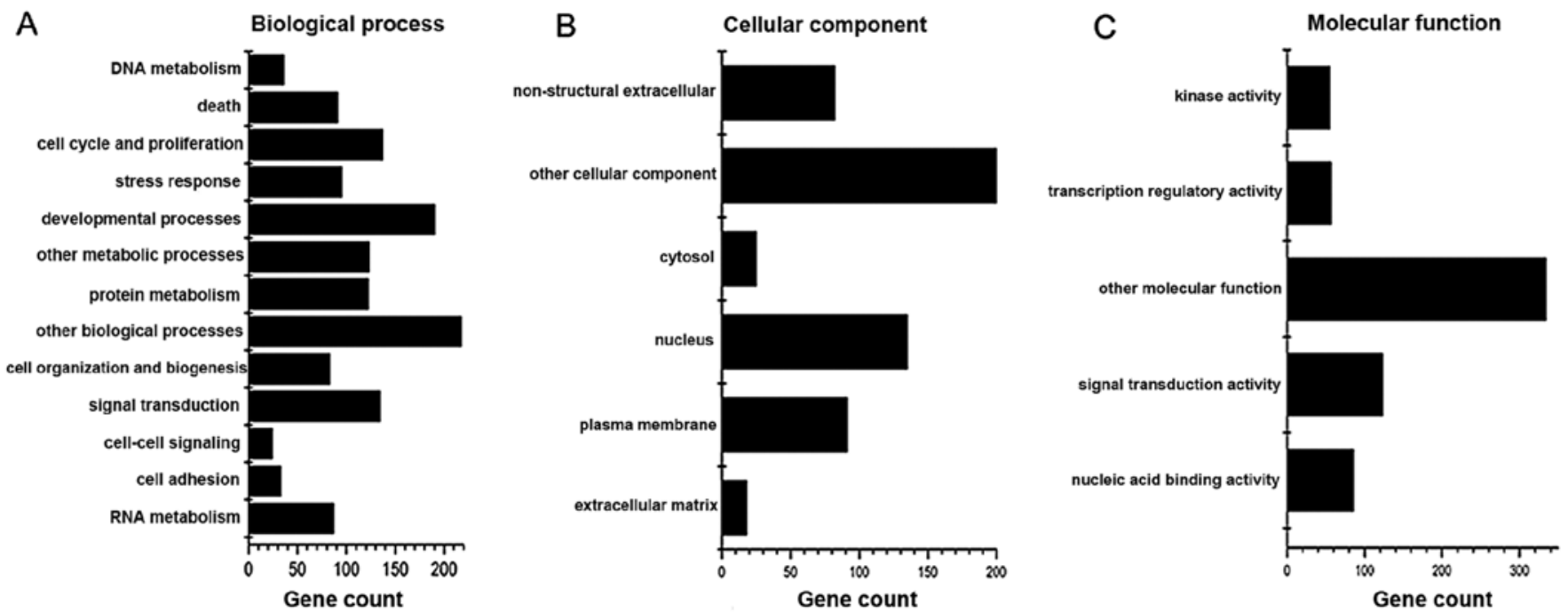

Figure 1. Gene ontology (GO) analysis of the RAS molecular network. GO enrichment sorted all data by Biological Process, Cellular Compartment and Molecular Function. GO analysis revealed that protein binding is the most prevalent molecular function of RAS interacting proteins, development, cell communication and signal transduction are the most common biological processes involved, and the gene products are active primarily in the cytoplasm, nucleus and plasma membrane.

$1 \mathrm{~h}$ at room temperature, and then analyzed by FACScan (Becton-Dickinson, USA). All experiments were performed in triplicate.

Statistical analysis. All tests were done using SPSS Graduate Pack 11.0 statistical software (SPSS, Chicago, IL, USA). Descriptive statistics including mean and SE, along with one-way ANOVA were used to determine significant differences. $\mathrm{P}<0.05$ was considered significant.

\section{Results}

Identification of Ras interaction genes. For RAS we queried Pubmed with: (K-RAS or N-RAS or H-RAS or RAS) and (cancer or tumor) and ("1980/01/01"[PDAT]: "2010/02/01"
[PDAT]). It led to the identification of 21,378 articles describing putative interactions between RAS and other genes. Sentences with the RAS gene, a proper interaction verb and another gene were selected and used for further analysis. A total of 439 genes that interacted with RAS were identified 393, 96 and 52 genes interacted with KRAS, NRAS and HRAS, respectively.

The biological function of the genes correlated with Ras. The gene ontology database was used. Our genes mapped to each node of GO database and the number of genes in these nodes were computed. The interaction genes of Ras were considered as a whole and displayed in three independent mode, for instance, biological process (Fig. 1A and Table II), cellular component (Fig 1B and Table III), molecular function (Fig. 1C and Table IV). 
Table II. Biological process.

\begin{tabular}{lrc}
\hline Term & Count & p-value \\
\hline 1 Cell cycle and proliferation & 137 & 0 \\
2 Death & 91 & 0 \\
3 DNA metabolism & 36 & 0 \\
4 Stress response & 95 & $5.36 \mathrm{E}-12$ \\
5 Developmental processes & 190 & $3.29 \mathrm{E}-11$ \\
6 Other metabolic processes & 123 & $5.46 \mathrm{E}-11$ \\
7 Protein metabolism & 122 & $5.70 \mathrm{E}-11$ \\
8 Other biological processes & 217 & $1.08 \mathrm{E}-08$ \\
9 Cell organization and biogenesis & 83 & $5.63 \mathrm{E}-08$ \\
10 Signal transduction & 134 & $1.56 \mathrm{E}-07$ \\
11 Cell-cell signaling & 24 & $2.22 \mathrm{E}-06$ \\
12 Cell adhesion & 33 & $1.83 \mathrm{E}-05$ \\
13 RNA metabolism & 87 & 0.002354 \\
\hline
\end{tabular}

Table III. Cellular component.

\begin{tabular}{lrc}
\hline Term & Count & p-value \\
\hline 1 Non-structural extracellular & 82 & $2.46 \mathrm{E}-11$ \\
2 Other cellular component & 203 & $5.34 \mathrm{E}-07$ \\
3 Cytosol & 25 & $6.21 \mathrm{E}-07$ \\
4 Nucleus & 135 & $4.75 \mathrm{E}-06$ \\
5 Plasma membrane & 91 & $4.25 \mathrm{E}-05$ \\
6 Extracellular matrix & 18 & 0.000108 \\
\hline
\end{tabular}

Table IV. Molecular function.

\begin{tabular}{lrc}
\hline Term & Count & p-value \\
\hline 1 Kinase activity & 55 & 0 \\
2 Transcription regulatory activity & 57 & $8.10 \mathrm{E}-09$ \\
3 Other molecular function & 334 & $9.80 \mathrm{E}-07$ \\
4 Signal transduction activity & 123 & $9.87 \mathrm{E}-07$ \\
5 Nucleic acid binding activity & 85 & 0.00043245 \\
\hline
\end{tabular}

Pathway and gene network analysis. KEGG pathway database was used to count each pathway enrichment p-value. We obtained a total of 128 signaling pathway. In addition, seventeen pathways (Table V) where identified whose corrected p-value was $<0.01$ and gene count was $<10$. Subsequently, thirteen cancers (Table VI) were related to this signaling pathway, most of them were human epithelial tumors.

The three results of ECrel, PPrel and GErel were integrated into the relationship between gene network. So we created a Ras-related gene network (Fig. 2A). Construction of the gene network of Ras-related genes revealed several Hub genes, or genes in which high connection exists giving these genes an
Table V. Signal pathway interrelated with Ras.

\begin{tabular}{|c|c|c|}
\hline Title & Count & $\mathrm{p}$-value \\
\hline 1 MAPK signaling pathway & 48 & 0 \\
\hline 2 ErbB signaling pathway & 29 & 0 \\
\hline 3 Toll-like receptor signaling pathway & 28 & $1.52 \mathrm{E}-12$ \\
\hline 4 Focal adhesion & 45 & $8.61 \mathrm{E}-13$ \\
\hline 5 P53 signaling pathway & 23 & $5.29 \mathrm{E}-12$ \\
\hline 6 Neurotrophin signaling pathway & 31 & $2.01 \mathrm{E}-11$ \\
\hline $7 \mathrm{~T}$ cell receptor signaling pathway & 26 & $5.29 \mathrm{E}-10$ \\
\hline 8 Jak-STAT signaling pathway & 29 & 2.64E-08 \\
\hline 9 Cell cycle & 24 & 4.27E-08 \\
\hline 10 Apoptosis & 20 & $1.58 \mathrm{E}-07$ \\
\hline 11 Adipocytokine signaling pathway & 15 & $7.15 \mathrm{E}-06$ \\
\hline 12 mTOR signaling pathway & 13 & 8.14E-06 \\
\hline 13 VEGF signaling pathway & 15 & 3.03E-05 \\
\hline 14 TGF- $\beta$ signaling pathway & 16 & 4.23E-05 \\
\hline 15 Insulin signaling pathway & 21 & $6.56 \mathrm{E}-05$ \\
\hline 16 Wnt signaling pathway & 19 & 0.00166 \\
\hline 17 Antigen processing and presentation & 13 & 0.00215 \\
\hline
\end{tabular}

Table VI. Cancers interrelated with Ras.

\begin{tabular}{|c|c|c|c|}
\hline \multicolumn{2}{|c|}{ Title } & \multirow{2}{*}{$\begin{array}{c}\text { Count } \\
108\end{array}$} & \multirow{2}{*}{$\frac{p \text {-value }}{2.65 \mathrm{E}-11}$} \\
\hline & Pathways in cancer & & \\
\hline 1 & Prostate cancer & 41 & 0 \\
\hline 2 & Colorectal cancer & 39 & 0 \\
\hline 3 & Pancreatic cancer & 38 & 0 \\
\hline 4 & Non-small cell lung cancer & 28 & 0 \\
\hline 5 & Glioma & 27 & 0 \\
\hline 6 & Endometrial cancer & 23 & 0 \\
\hline 7 & Chronic myeloid leukemia & 32 & $4.07 \mathrm{E}-13$ \\
\hline 8 & Small cell lung cancer & 26 & $1.67 \mathrm{E}-12$ \\
\hline 9 & Melanoma & 30 & $5.32 \mathrm{E}-12$ \\
\hline 10 & Bladder cancer & 27 & $6.28 \mathrm{E}-12$ \\
\hline 11 & Renal cell carcinoma & 22 & $3.71 \mathrm{E}-11$ \\
\hline 12 & Thyroid cancer & 14 & $2.21 \mathrm{E}-10$ \\
\hline 13 & Acute myeloid leukemia & 18 & $2.46 \mathrm{E}-09$ \\
\hline
\end{tabular}

influential role in network stability. Generally, the Hub genes which would influence the majority of genes were central to gene regulation, therefore, more important than other genes. Finally, according to their overlaid relationship, we chose top nine (PIK3CA, MDM2, CCND1, EGFR, JUN, MYC, VEGFA, ERK1 and ERK2) whose p-value was $<0.05$ as the Hub genes (Fig. 2B). EGFR and VEGFA which were in the upper stream of some pathways could activate the signal transduction pathway. Other genes were all in the middle element of the Ras signaling pathway. 
A

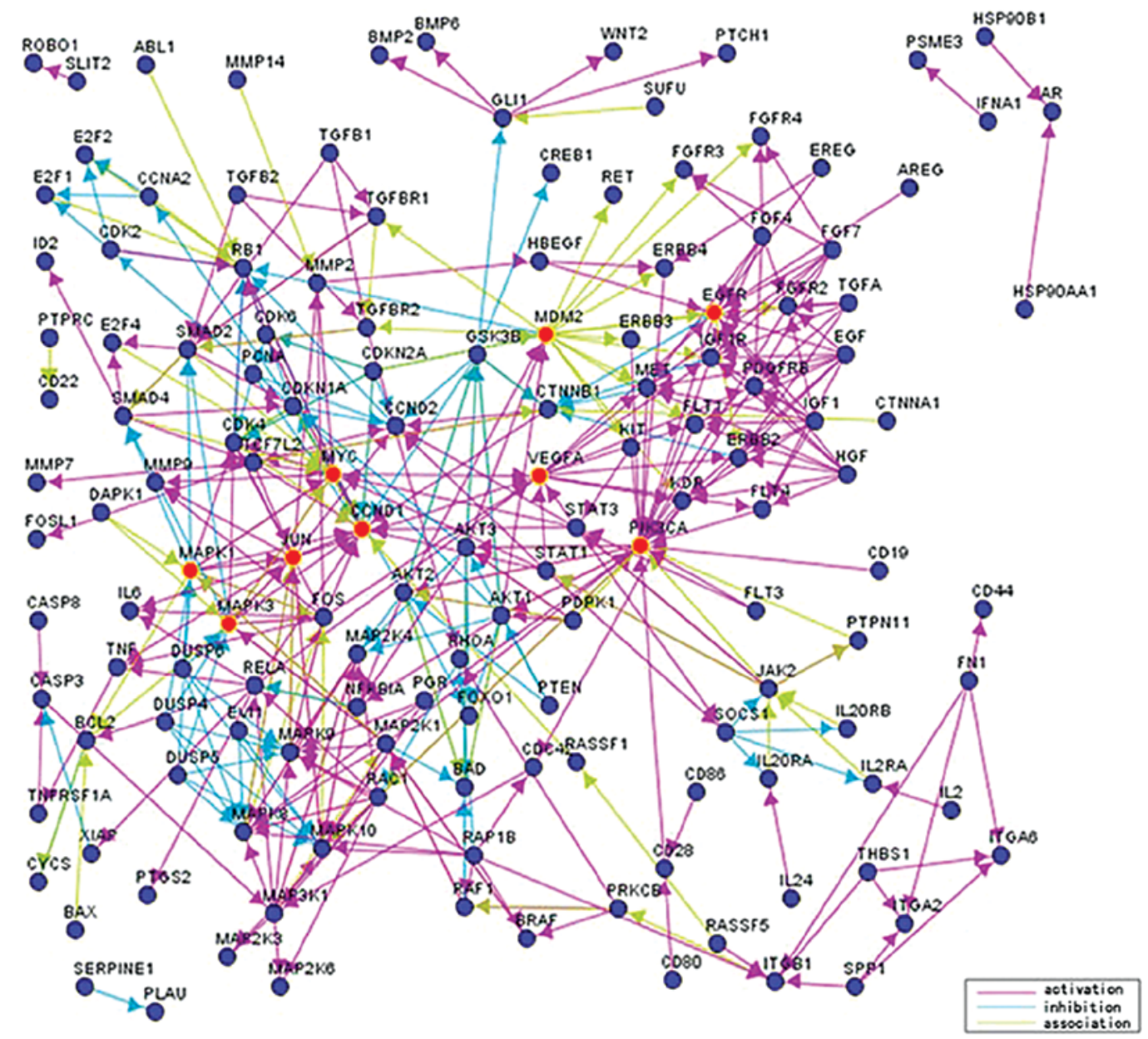

B

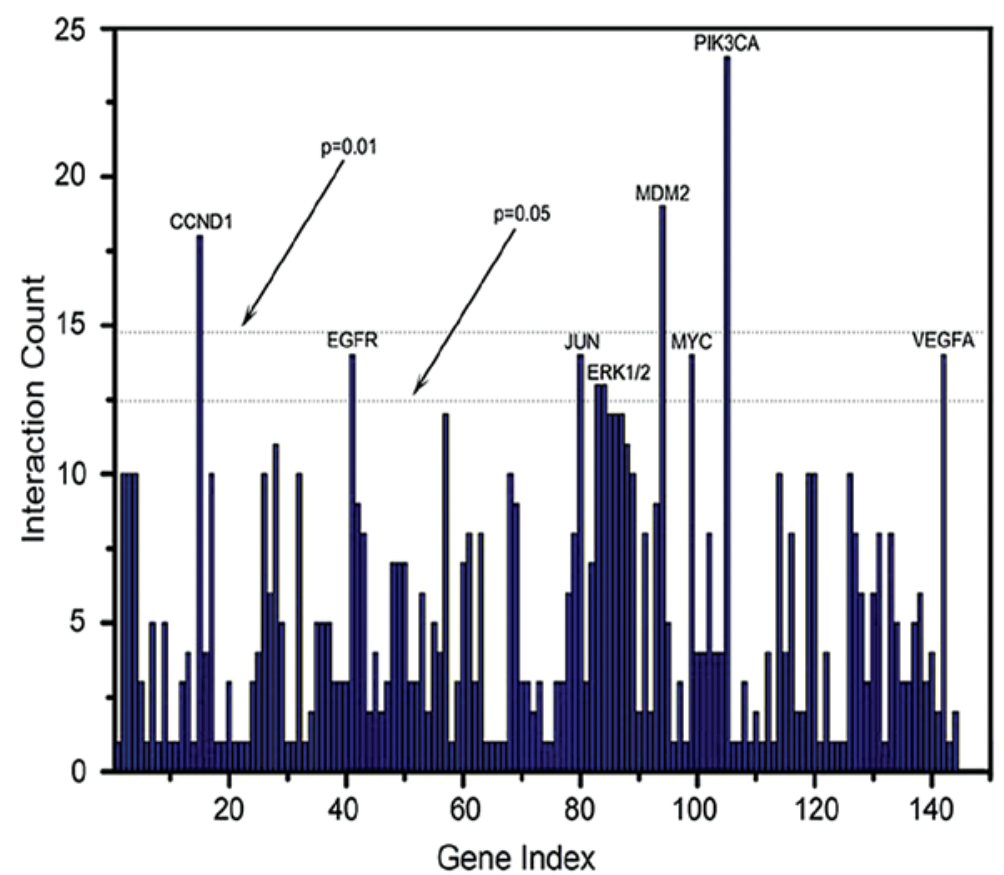

Figure 2. Visualization of RAS-related gene interaction network. (A) Visualization of the RAS related gene network. The network was visualized with Medusa software. The pink connecting wire is activation, the blue is inhibition and the yellow is association. The red round symbols represent Ras-related Hub genes which are considered the core of gene regulation. (B) Connectivity analysis of the RAS-related gene network. Connectivity analysis was performed using the Search Tool for the Retrieval of Interacting Genes/Proteins to generate the RAS related genes knowledge-driven network, as described in Materials and methods. Analysis revealed PIK3CA, MDM2, CCND1, EGFR, JUN, MYC, VEGFA, ERK1 and ERK2 are important Hub genes in the RAS network, with mean frequency counts $>13(\mathrm{P}<0.05)$. 


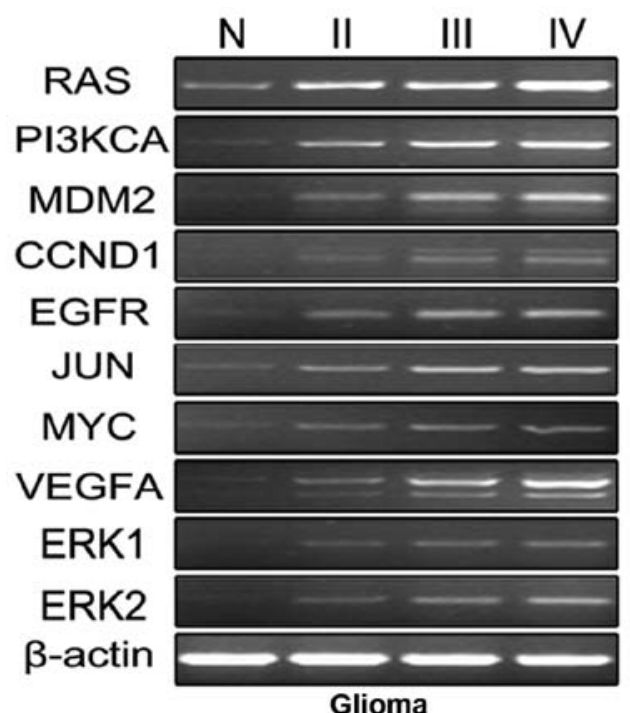

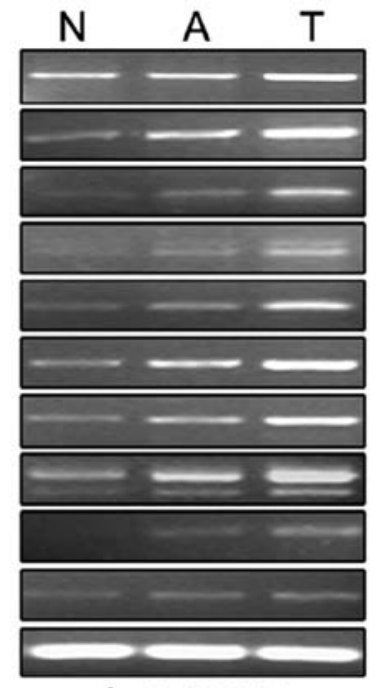

Lung cancer

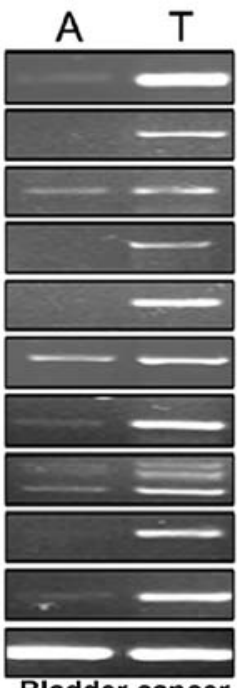

Bladder cancer

Figure 3. The relationship between RAS and other Hub genes. RT-PCR analysis was used to detect the expression of Ras and Hub genes in the tissues of glioma, lung cancer and bladder cancer. In the different pathological grades their expression contrast was remarkable.

The expression of Ras and Hub genes in epithelial tumors. To explore the expression of RAS in the epithelial tumors, we investigated glioma, lung cancer and bladder cancer tissue samples by semi-quantitative RT-PCR analysis. The expression of RAS mRNA was markedly higher in three tumor tissue specimens. In glioma, compared to normal tissue and low grade gliomas (II), the mRNA level of Ras expression in higher grade gliomas (III and IV) was remarkably higher. Bladder cancer and lung cancer tissue specimens, contrasted with adjacent non-cancer tissue, the expression of Ras was significantly stronger in the tumor tissues (Fig. 3). Further, the levels of Hub genes, including PIK3CA, MDM2, CCND1, EGFR, JUN, MYC, VEGFA, ERK1 and ERK2 were higher in their respective tumor tissue.

Inhibition of activated Ras suppresses cell malignancy and inhibits multiple oncogenes in human epithelial tumors. To study the role of biological behavior of Ras in human epithelial tumor cells, we introduced the Ras inhibitor FTS to inhibit its activity. We examined the effect of FTS on U87, A549 and T24 cell proliferation with different doses. After $48 \mathrm{~h}$, counting of viable cells showed that the inhibitor induced a dose-dependent decrease in cell number (Fig. 4A). In addition, when the survival rates of cells were $\sim 50 \%$, we considered that the suitable concentration of FTS was 70, 55 and $50 \mu \mathrm{M}$, respectively. The epithelial tumor cells from each group were treated with FTS for $48 \mathrm{~h}$, and then the cell cycle was measured by flow cytometry. Cell cycle analysis indicated that FTS significantly caused G1 phase arrest in the epithelial tumor cells compared with the blank group and the negative group (Fig. 4B). To investigate the biological function of Ras on the apoptosis of the epithelial tumor cells, as shown in Fig. 4C, compared with blank and negative controls, statistically significant more apoptotic cells were found in epithelial tumor cells in activated $\operatorname{Ras}(\mathrm{P}<0.05)$.

The biological behavior of Hub genes involve survival/ apoptosis, proliferation/cell cycle, differentiation and tran- scription, and appears to play a central role in the etiology and progression of solid tumors. Western blot analysis was used to investigate the relationship between Ras and Hub genes. Inhibition of activated Ras resulted in decreased expression of Hub genes in U87, A549 and T24 cells, respectively (Fig. 5).

\section{Discussion}

In the present study, we confirmed the biological function of interaction genes of Ras through the bioinformatic analysis. Top nine $(\mathrm{p}<0.05)$ Hub genes were identified and the pathway and gene network of Ras were established. In addition, then we showed that the expression of Ras and Hub genes in three human epithelial tumor tissues and suppression of activated Ras led to human epithelial tumor cell lines growth suppression and apoptosis increase in vitro. Furthermore, we demonstrated suppression of activated Ras inhibited the Ras pathway by blocking multiple oncogenic targets in human epithelial tumor cells.

Malignant tumors, are a major problem of human health. The cells escape the normal growth regulation mechanism by controlling the expression of several genes (7-9). Recent studies have showed that approximately one third of all human cancers, including glioma, lung cancer and bladder cancer, express a constitutively active oncogenic Ras. Abnormal activation of Ras triggers series of physiopathological changes and oncogenesis (10). In summary, the relationship between Ras and tumors remains unclear. Gene/protein interaction relationship provides us with a comprehensive understanding of biological processes (11). The accumulation of a large number of biomedical literature is an untapped biological information database for the study of gene/protein interaction networks to provide a reliable data source $(12,13)$. Here, we show the tumor biological signaling networks related to the development of Ras-related genes and found Ras-related gene expression to participate in biological processes, cellular components, 


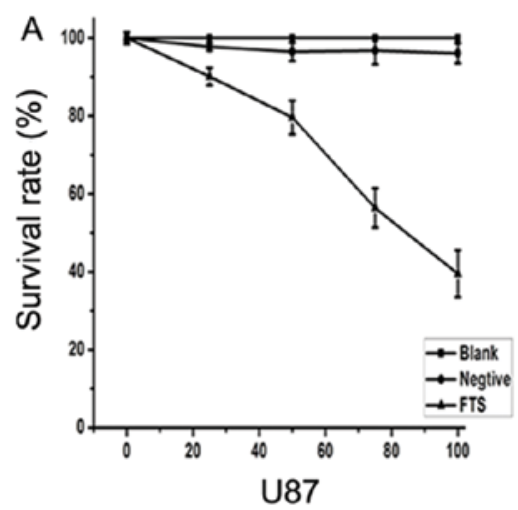

B
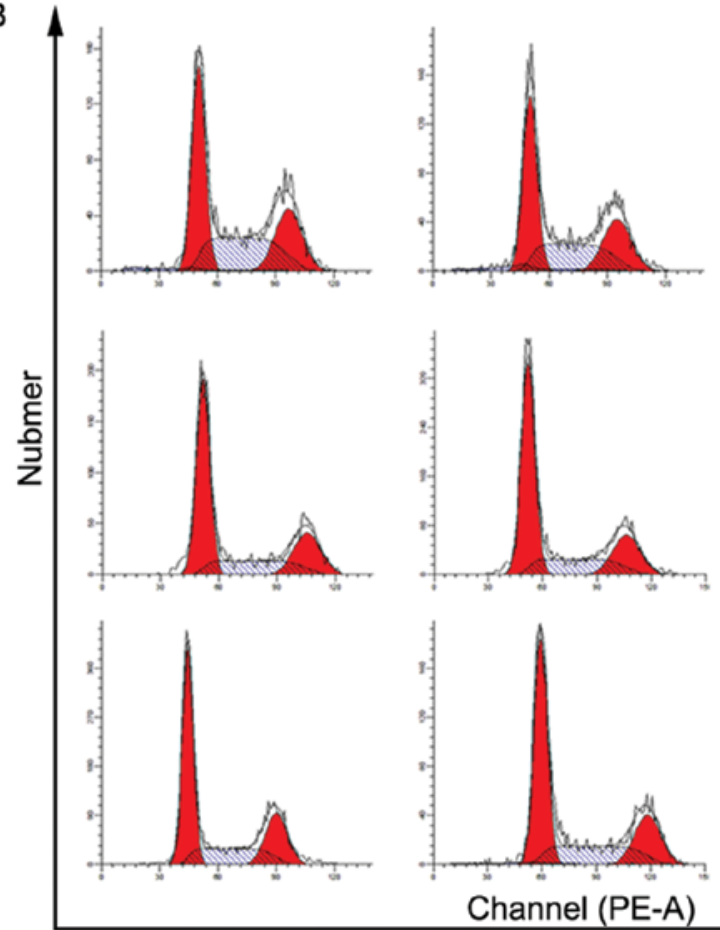

C

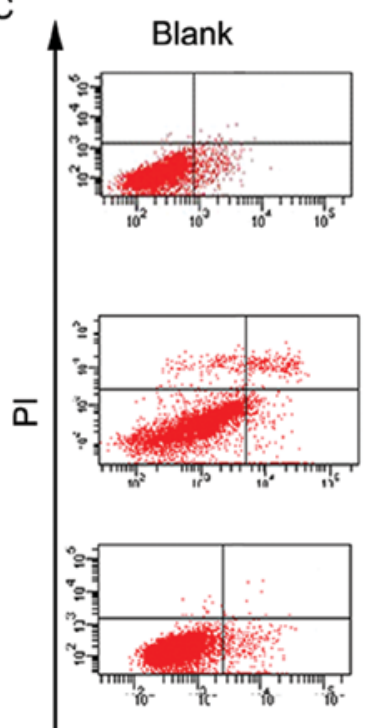

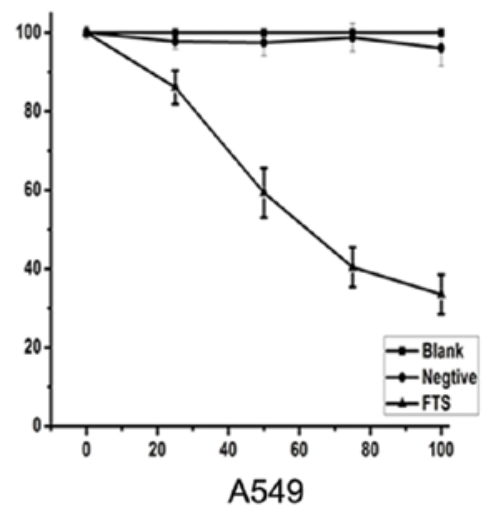

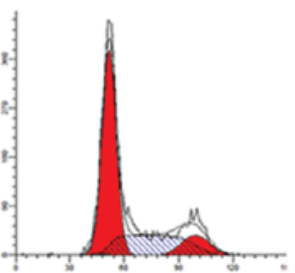

U87
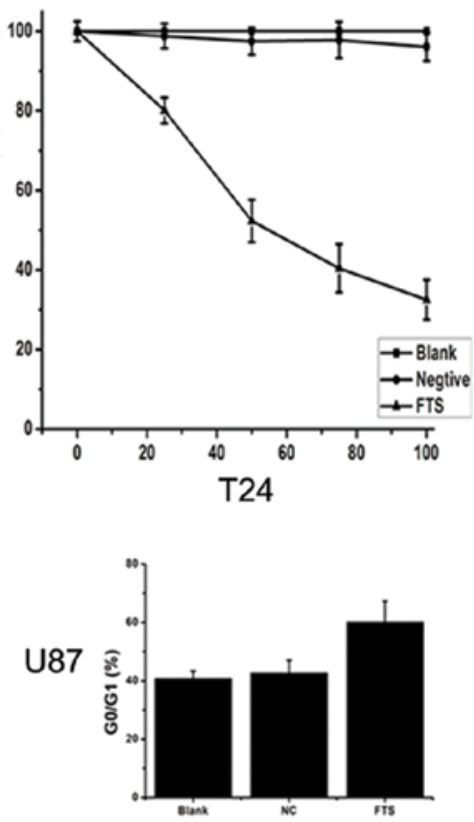

A549

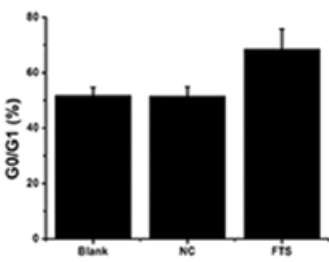

T24

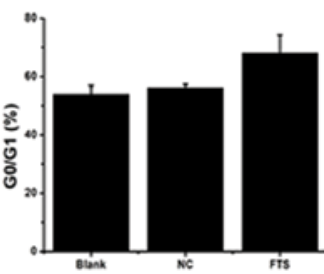

U87

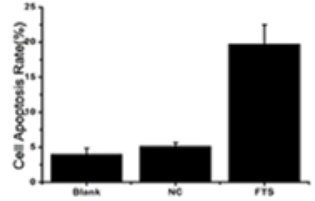

A549

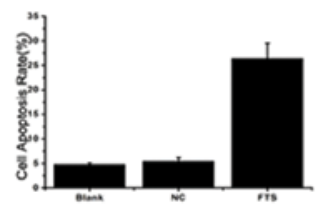

T24

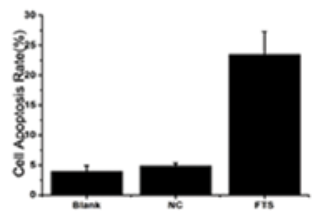

Figure 4. Inhibition of Ras suppressed cell proliferation in human glioma, lung cancer and bladder cancer cell lines. (A) The cell growth inhibition rates of U87, A549 and T24 cells after treatment with FTS at different doses. The survival rate in the control group is presented as $100 \%$. The data are presented as the mean \pm SD. (B) The effect of inhibition of Ras on cell cycle in tumor cell lines. The cell cycle was analyzed on FACScan by flow cytometry $48 \mathrm{~h}$ after the FTS treatment. Tumor cell were harvested and fixed. Percentage of cells in G1, G2 and S phases are presented as histograms. Each independent experiment was done three times. (C) FTS induced cell apoptosis in some human tumor cell lines. U87, A549 or T24 cells were treated with FTS. After 48-h treatment, cells were harvested and double stained for Annexin V and propidium iodide and analyzed by flow cytometry. Early apoptotic cells are Annexin $\mathrm{V}^{+} / \mathrm{PI}^{-}$, late apoptotic cells are Annexin $\mathrm{V}^{+} / \mathrm{PI}^{+}$, necrotic cells are Annexin $\mathrm{V}^{-} / \mathrm{PI}^{+}$and healthy cells are Annexin $\mathrm{V}^{-} / \mathrm{PI}^{-}$. A representative experiment of the three performed is shown. 


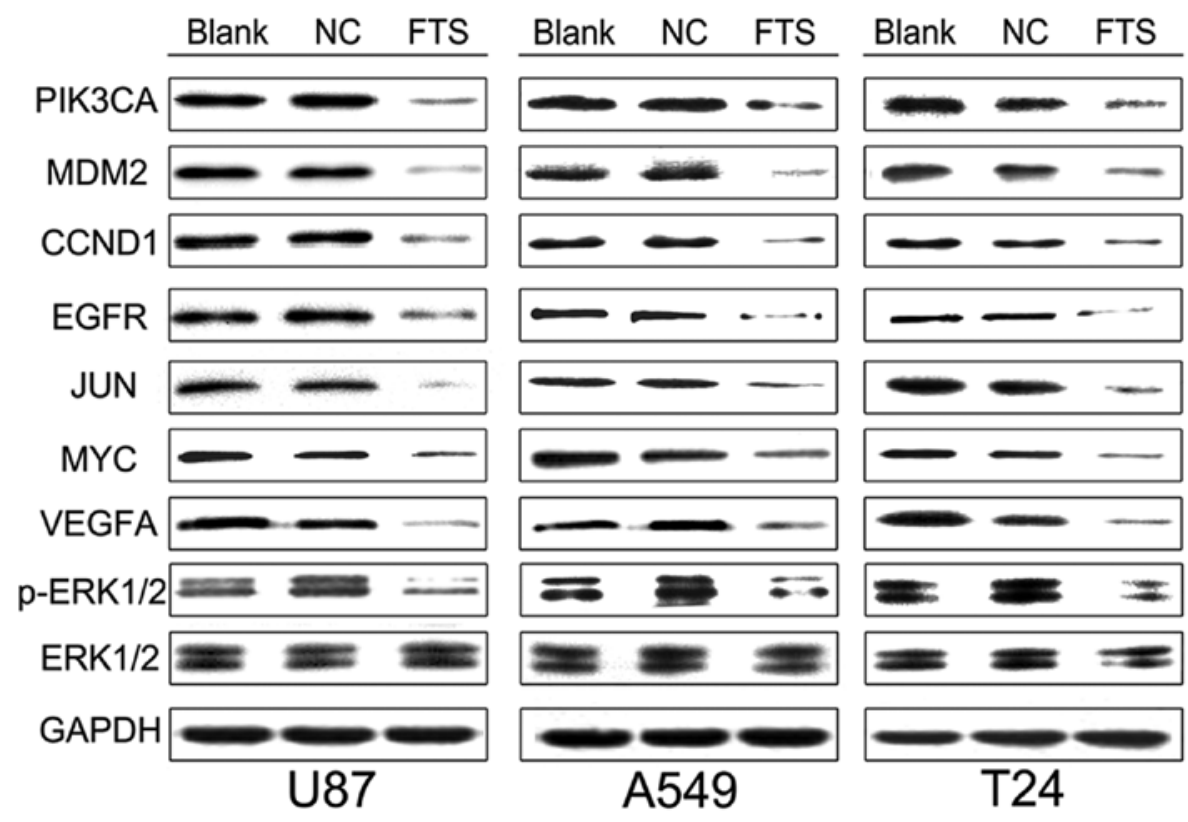

Figure 5. Ras regulates the Hub gene expression via inhibiting the Ras gene. Western blot analysis showed that the Hub gene protein level was significantly downregulated, except ERK1/2, when FTS treated. However, the level of phosphorylated ERK1/2 decreased.

molecular functions, signal transduction pathways, cancer occurrence and development. Study of gene/protein interactions found PIK3CA, MDM2, CCND1, EGFR, JUN, MYC, VEGFA, MAPK1/3 in the Ras gene network. They are involved in the MAPK signaling pathway. The Hub gene stability of the network plays an important role in gene regulation.

As an answer to the question of whether inhibition of activated Ras led to growth suppression of the human epithelial tumor cell lines, the increased apoptosis was associated with Ras downstream signaling. The Ras inhibitor (FTS) was included in our research. FTS is a synthetic farnesylcysteine mimic. It can not only prevent Ras from anchoring to the membrane, but also prevents Ras from combining with its molecular chaperone by removing it from the membrane (14-16). In addition, FTS promotes the degradation of Ras suspending it in the cytoplasm to reduce the level of activated Ras, which results in reduction of cancer incidence. In this experiment, our results confirmed repression of activated Ras by FTS inducing a reduction of most of the Hub gene proteins. Most of these Hub genes are transcription factors and sometimes kinases, and can regulate proliferation, differentiation, apoptosis, tumorigenesis, and transformation.

Deregulation of the Ras pathway leads to complex multiple signaling cascades that contribute to human malignancies $(17,18)$. Ras is therefore considered an appropriate target for cancer therapy (19). In light of the complexity of the deregulated Ras pathway, it is important to decipher at the molecular level the response of cancer cells to Ras inhibitors that would reregulate it. Recent research (20-22) has shown that liver neoplasms are delayed in rats if they were fed with identical concentration of FTS every day. The University of Texas MD Anderson Cancer Center has implemented a program of phase I clinical trials of FTS to treat pancreatic cancer and demonstrated that the drug has no overlapping pharmacokinetics with other chemotherapeutic drugs (20). Balasis et al
(23) have acquired great achievements in treating breast cancer combined with FTS in rats.

\section{Acknowledgements}

This study was supported by grants from the National High Technology Research and Development Program of China (863) (2012AA02A508), National Natural Science Foundation of China (91229121, 81272792, 81172389, 81372709, 81302185, 81101901), Jiangsu Province Natural Science Foundation (BK2011847 and 20131019), Jiangsu Province Key Provincial Talents Program (RC2011051), Jiangsu Province Key Discipline of Medicine (XK201117), Jiangsu Provincial Special Program of Medical Science (BL2012028), and Program for Development of Innovative Research Team in the First Affiliated Hospital of NJMU, and the Priority Academic Program Development of Jiangsu Higher Education Institutions (PAPD).

\section{References}

1. Zhao P, Wang C, Fu Z, et al: Lentiviral vector mediated siRNA knock-down of hTERT results in diminished capacity in invasiveness and in vivo growth of human glioma cells in a telomere length-independent manner. Int J Oncol 31: 361-368, 2007.

2. Schwartzbaum JA, Fisher JL, Aldape KD and Wrensch M: Epidemiology and molecular pathology of glioma. Nat Clin Pract Neurol 2: 494-503, quiz 1 p following 516, 2006.

3. Ellis RW, Defeo D, Shih TY, et al: The p21 src genes of Harvey and Kirsten sarcoma viruses originate from divergent members of a family of normal vertebrate genes. Nature 292: 506-511, 1981.

4. Der CJ, Krontiris TG and Cooper GM: Transforming genes of human bladder and lung carcinoma cell lines are homologous to the ras genes of Harvey and Kirsten sarcoma viruses. Proc Natl Acad Sci USA 79: 3637-3640, 1982.

5. Shimizu K, Goldfarb M, Suard Y, et al: Three human transforming genes are related to the viral ras oncogenes. Proc Natl Acad Sci USA 80: 2112-2116, 1983.

6. Robinson JP, VanBrocklin MW, Guilbeault AR, Signorelli DL, Brandner S and Holmen SL: Activated BRAF induces gliomas in mice when combined with Ink4a/Arf loss or Akt activation. Oncogene 29: 335-344, 2010. 
7. Nandan MO and Yang VW: An update on the biology of RAS/ RAF mutations in colorectal cancer. Curr Colorectal Cancer Rep 7: 113-120, 2011

8. Abel TW, Clark C, Bierie B, et al: GFAP-Cre-mediated activation of oncogenic K-ras results in expansion of the subventricular zone and infiltrating glioma. Mol Cancer Res 7: 645-653, 2009.

9. Holmen SL and Williams BO: Essential role for Ras signaling in glioblastoma maintenance. Cancer Res 65: 8250-8255, 2005.

10. Hagemann C, Gloger J, Anacker J, et al: RAF expression in human astrocytic tumors. Int J Mol Med 23: 17-31, 2009.

11. Khan SH, Ahmad F, Ahmad N, Flynn DC and Kumar R: Proteinprotein interactions: principles, techniques, and their potential role in new drug development. J Biomol Struct Dyn 28: 929-938, 2011.

12. Yue X, Lan F, Yang W, et al: Interruption of beta-catenin suppresses the EGFR pathway by blocking multiple oncogenic targets in human glioma cells. Brain Res 1366: 27-37, 2010.

13. Braccini L, Ciraolo E, Martini M, et al: PI3K keeps the balance between metabolism and cancer. Adv Biol Regul 52: 389-405, 2012.

14. Marciano D, Ben-Baruch G, Marom M, Egozi Y, Haklai R and Kloog Y: Farnesyl derivatives of rigid carboxylic acids - inhibitors of ras-dependent cell growth. J Med Chem 38: 1267-1272, 1995.

15. Haklai R, Weisz MG, Elad G, et al: Dislodgment and accelerated degradation of Ras. Biochemistry 37: 1306-1314, 1998.
16. Rotblat B, Ehrlich M, Haklai R and Kloog Y: The Ras inhibitor farnesylthiosalicylic acid (Salirasib) disrupts the spatiotemporal localization of active Ras: a potential treatment for cancer. Methods Enzymol 439: 467-489, 2008.

17. Overmeyer JH and Maltese WA: Death pathways triggered by activated Ras in cancer cells. Front Biosci 16: 1693-1713, 2011.

18. Margarit SM, Sondermann H, Hall BE, et al: Structural evidence for feedback activation by Ras.GTP of the Ras-specific nucleotide exchange factor SOS. Cell 112: 685-695, 2003.

19. McCormick F: Cancer therapy based on oncogene addiction J Surg Oncol 103: 464-467, 2011.

20. Schneider-Merck T, Borbath I, Charette N, et al: The Ras inhibitor farnesylthiosalicyclic acid (FTS) prevents nodule formation and development of preneoplastic foci of altered hepatocytes in rats. Eur J Cancer 45: 2050-2060, 2009.

21. Roskoski RJ: ERK1/2 MAP kinases: structure, function, and regulation. Pharmacol Res 66: 105-143, 2012.

22. Ferro E and Trabalzini L: RalGDS family members couple Ras to Ral signalling and that's not all. Cell Signal 22: 1804-1810, 2010.

23. Balasis ME, Forinash KD, Chen YA, et al: Combination of farnesyltransferase and Akt inhibitors is synergistic in breast cancer cells and causes significant breast tumor regression in ErbB2 transgenic mice. Clin Cancer Res 17: 2852-2862, 2011. 